\title{
Specific suppression of microgliosis cannot circumvent the severe neuropathology in peroxisomal $\beta$-oxidation-deficient mice
}

L. Beckers ${ }^{1}$, S. Stroobants ${ }^{2}$, S. Verheijden ${ }^{1}$, B. West ${ }^{3}$, R. D'Hooge ${ }^{2}$, M. Baes $^{1}$

${ }^{1} \mathrm{KU}$ Leuven - University of Leuven, Department of Pharmaceutical and Pharmacological Sciences, Cell Metabolism, B-3000 Leuven, Belgium; ${ }^{2} \mathrm{KU}$ Leuven - University of Leuven, Faculty of Psychology and Educational Sciences, Biological Psychology, B-3000 Leuven, Belgium, ${ }^{3}$ Plexxikon Inc., Berkeley, CA 94710, USA

Running title:

PLX5622 treatment of $M f p 2^{-/-}$mice

\section{Keywords:}

Microglia; peroxisomal beta-oxidation; neuroinflammation; cognition; neuronal transmission; behavior 


\section{ABSTRACT}

An important hallmark of various neurodegenerative disorders is the proliferation and activation of microglial cells, the resident immune cells of the central nervous system (CNS). Mice that lack multifunctional protein-2 (MFP2), the key enzyme in peroxisomal $\beta$-oxidation, develop excessive microgliosis that positively correlates with behavioral deficits whereas no neuronal loss occurs. However, the precise contribution of neuroinflammation to the fatal neuropathology of MFP2 deficiency remains largely unknown. Here, we first attempted to suppress the inflammatory response by administering various anti-inflammatory drugs but they failed to reduce microgliosis. Subsequently, Mfp2 $^{-1-}$ mice were treated with the selective colony-stimulating factor 1 receptor (CSF1R) inhibitor PLX5622 as microglial proliferation and survival is dependent on CSF1R signaling. This resulted in the elimination of more than $95 \%$ of microglia from control mice but only $70 \%$ of the expanded microglial population from $\mathrm{Mfp}^{-/-}$mice. Despite microglial diminution in $\mathrm{Mfp}^{-/}$brain, inflammatory markers remained unaltered and residual microglia persisted in a reactive state. CSF1R inhibition did not prevent neuronal dysfunction, cognitive decline and clinical deterioration of $\mathrm{Mfp}^{-/}$mice. Collectively, the unaltered inflammatory profile despite suppressed microgliosis, concurrent with persevering clinical decline strengthens our hypothesis that neuroinflammation importantly contributes to the Mfp2-phenotype. 


\section{INTRODUCTION}

Peroxisomes and peroxisomal $\beta$-oxidation in particular are essential for the formation and maintenance of the CNS as deficits cause devastating neurological diseases in men and mice [1-3]. However, the precise molecular mechanisms remain elusive. The most common neurometabolic disorder caused by peroxisomal $\beta$-oxidation deficiency is X-linked adrenoleukodystrophy [4], characterized by myelopathy and, in a subgroup of patients, by fatal cerebral inflammatory demyelination. Although in the latter microglia seem to play a pivotal role in disease progression $[5,6]$, this cannot be further investigated in the corresponding mouse model $\left(A b c d 1^{-/}\right)$as only spinal cord pathology develops [4]. We have generated and analyzed mouse models with constitutive and cell type-selective deletion of multifunctional protein-2 (MFP2, also known as D-bifunctional protein and HSD17B4), the pivotal enzyme of peroxisomal $\beta$-oxidation [7-9]. In contrast to Abcd1- mice, Mfp $2^{-/-}$mice develop excessive neuroinflammation from the age of 6 iveek that worsens with age $[7,9,10]$. Mfp2 ${ }^{-1}$ rese xhibit progressive motor deficits and lethargy and die before the age of 6 months $[9,10]$. This phenotype does not correspond with the severe neurodevelopmental pathology of patients with total ablation of MFP2 [11], but rather mimics milder mutations in patients that give rise to neurological anomalies including ataxia, leukodystrophy and vision/hearing problems [11-13].

The microgliosis in $\mathrm{Mfp}^{--}$mice, characterized by increased microglial numbers and morphological transformation, exclusively depends on the proliferation of resident cells [7]. The expansion of the microglial population positively correlates with the severity of neuronal dysfunction and clinical deterioration (Beckers and Baes, unpublished data) but the underlying sequence of pathological events is poorly understood. Microgliosis is confined to gray matter areas and Mfp2-- microglia surround neurons, indicating a pathological role of disturbed microglia-neuron communication in MFP2 deficiency. Despite affected neuronal transmission and clinical deterioration in $\mathrm{Mfp}^{---}$mice, there is no obvious loss of neuron numbers ([7], Beckers and Baes, unpublished data).

An outstanding question is how chronic microgliosis and aberrant phenotype of $M f p 2^{-/-}$microglia impact on neuronal dysfunction and murine behavioral deficits. Therefore, we first attempted to suppress neuroinflammation by using agents that were successfully used in neurodegenerative diseases to reduce microglial reactivity and pro-inflammatory markers i.e. minocycline $[14,15]$, pioglitazone $[16,17]$ and 5-androsten-3 $\beta, 17 \beta$-diol (ADIOL) [18]. Because these treatments failed to improve the inflammatory state of the brain and the clinical deterioration of the mice, we subsequently tried to specifically eradicate microglia from $M f p 2^{-/}$brain. It was previously shown that microglia in the adult brain are utterly dependent on CSF1R signaling for their survival and maintenance [19, 20]. Accordingly, administration of CSF1R inhibitors that cross the blood-brain barrier were shown to cause brain-wide elimination (90-98\%) of microglia within 7 days of treatment without altering circulating monocyte numbers [21, 22]. Moreover, eliminating microglia in neurodegenerative diseases such as Alzheimer's disease $(A D)$ prevents neuronal loss and improved performance in memory and behavioral tasks [2328]. Specific microglial depletion has never been tested in neurometabolic or non-neurodegenerative disorders. Hence, we treated $\mathrm{Mfp}^{-/}$and control mice for 10 weeks with the CSF1R inhibitor PLX5622. We found that this compound effectively eliminated all microglia from control brain but only significantly reduced and did not obliterate the activated microglia from $M f p 2^{-/}$brain. Pharmacological targeting of 
CSF1R could not prevent the clinical deterioration nor improve neuronal dysfunction and cognition of $\mathrm{Mfp}^{-/-}$mice.

\section{MATERIALS AND METHODS}

\section{Mouse breeding}

The generation of $\mathrm{Mfp}^{-/}$mice has been described [29] but mice were now bred into a C57BL/6J background for seven generations. As we did not detect differences between wild type and heterozygous mice in our previous investigations, they were both used as controls. Genotyping was performed on tail DNA. All mice were bred in the animal housing facility of the KU Leuven, had ad libitum access to water and standard rodent food, and were kept on a 12-hour light and dark cycle. All experiments were conducted in accordance with "Guidelines for Care and Use of Experimental Animals" and fully approved by the Research Ethical committee of the KU Leuven (\#190/2012, $\# 181 / 2015)$.

\section{Administration of anti-inflammatory drugs}

Minocycline (50 mg/kg, Abc chemicals, Wouters-Brakel, Belgium) or PBS was administered by daily intraperitoneal injection [30] from the age of 9 weeks till the age of 15 weeks. Pioglitazone hydrochloride (sc-204848, Santa Cruz Biotechnology) was formulated with normal mouse chow and gelatin at 240 ppm. The same diet but containing vehicle was used as control. Briefly, normal chow was pulverized and thoroughly mixed with either pioglitazone hydrochloride or vehicle. Gelatin was dissolved in hot water (below boiling point) and mixed with pulverized chow. The food chunks were kept at $4^{\circ} \mathrm{C}$ for at least 2 hours. Control and $M f p 2^{-/}$mice ( $n=7-8 /$ group) were fed the diets from the age of 10 weeks till the age of 15 weeks. Every 2-3 days, chow was renewed and food intake was measured. Clinical scores were weekly determined throughout the treatment period and prior to sacrifice.

\section{Administration of PLX5622}

PLX5622 was provided by Plexxikon Inc. and formulated in AIN-76A standard chow by Research Diets Inc. at $1200 \mathrm{ppm}$. The same diet containing vehicle was used as control. Control and Mfp2-- mice $(\mathrm{n}=$ 9/group) were fed the diets from the age of 6 weeks till the age of 16 weeks. Clinical scores were determined throughout the treatment period and behavioral studies were conducted prior to sacrifice.

\section{Murine behavioral studies}

Auditory brainstem response test. Brainstem auditory-evoked potentials (BAEPs) were recorded on a Myos 4 plus digital EMG/EP recorder (Schwarzer, Munich, Germany). Mice were anesthetized with Nembutal (6 mg/ml i.p. at $1 \%$ body weight). A needle electrode was placed subcutaneously behind each ear and referenced to a common electrode near the base of the tail; a fourth electrode, also placed near the base of the tail, was used as a ground. Robust five-peak tracings were obtained by averaging 2000 responses evoked by 85-86 dB clicks [measured with a Brüel \& Kjær (Norcross, GA) sound 
intensity meter] emitted by a speaker placed $1 \mathrm{~cm}$ in front of the animal's head. For each of the tracings, latencies of the first five peaks (numbered I through $\mathrm{V}$ ) were measured as well as interpeak latencies IIII, III-V, and I-V. In addition, mean amplitudes of all peaks were measured.

Exploration by Open Field (OF) assessment. OF exploration was tested in a brightly illuminated $50 \mathrm{~cm}$ $\times 50 \mathrm{~cm}$ square arena subsequent to $30 \mathrm{~min}$ of dark adaptation. Movements in the arena were videotracked for $10 \mathrm{~min}$. Total path length, distance to the center and number of corner entries were assessed.

Passive Avoidance (PA) learning. PA learning was examined in a cage consisting of a light and a dark compartment containing a grid floor [31]. After 30 min adaptation to the dark, the mouse was placed in the light compartment for a training trial. After $5 \mathrm{~s}$, the dark compartment was opened and step-through latency was manually recorded. When all paws were placed on the grid floor, a mild electric footshock $(0.2 \mathrm{~mA}, 2 \mathrm{~s})$ was applied. Retention was tested $24 \mathrm{~h}$ later in the dark-adapted mouse, and latency to enter the dark compartment was measured up to a 300-s cut-off value.

Clinical score. Progression of disease was assessed and scored based on the severity of typical clinical symptoms including ataxia, gait disturbance, weakness, alertness/activity, cataract and kyphosis. Kyphosis is a condition in which the thoracic spine has an excessive outward curvature, causing hunching of the back. Scores from 0 to 5 were assigned according to severity of each symptom. A score of 0 was given to mice that were normal. A higher score was given if the symptom was more severe. The clinical score value is the sum of all scores from distinct symptoms.

Grip strength measurement. The grip strength test measures either the forepaw strength or a combined forepaw and hind paw strength. Mice were allowed to grasp the grip strength meter grid with their forepaws only, and were then gently pulled from the base of the tail until they release the grip. The grip strength meter measures the maximum force applied to the meter. This was repeated 5 times per mouse. Afterwards, grip strength of combined forepaw and hind paw strength were measured by repeating the protocol but placing the mouse with four paws on the grid before pulling it gently from the base of the tail.

Inverted grid test. The inverted grid test or four limb hang test is a test of combined forepaw and hind paw strength. Mice are placed on a wire grid which is then inverted. The latency to fall is recorded with a time limit of $300 \mathrm{~s}$. In general, normal mice are able to remain on the inverted grid for at least $300 \mathrm{~s}$. Mice that fall off the grid before the time limit of $300 \mathrm{~s}$ were directly given one more try. Mice that hang for the $300 \mathrm{~s}$ limit were placed back in the cage.

\section{Immunohistochemistry}

Mice were anesthetized with a mix of Dormitor $(1 \mathrm{mg} / \mathrm{kg})$ and Nimatek $(75 \mathrm{mg} / \mathrm{kg})$. Tissue processing and IHC staining were performed as described $[9,32,33]$. Briefly, mice were perfused transcardially 
with PBS ( $\mathrm{pH}$ 7.4) followed by $4 \%$ paraformaldehyde (PFA). Brains were isolated, post-fixed with $4 \%$ PFA overnight, and kept in $70 \%$ ethanol prior to paraffin embedding. Routinely, paraffin sections ( $7 \mu \mathrm{m})$ were used for immunofluorescent stainings. The following primary antibodies were used: polyclonal rabbit anti-IBA1 (1:500; Wako D19-19741), rat anti-F4/80 (1:500; Serotec, Oxford, UK). After incubation with primary antibodies overnight at room temperature HRP-labeled secondary antibodies (1:200) were applied for 1 hour, followed by fluorescent labeling with a cyanine 2 (FITC) TSA kit (Perkin Elmer Life sciences, Boston, USA). When double immunolabeling was performed, sets of primary and secondary antibodies were applied sequentially. As second fluorescent labels, cyanine 3 TSA kits (Perkin-Elmer) were used. Images were acquired with a motorized inverted IX-81 microscope connected to a CCDFV2T digital camera (Olympus, Aartselaar, Belgium) and processed with LSM Image browser software (Zeiss, Germany).

\section{Quantification of IHC staining}

Cell number quantification. Microglia cell numbers were quantified on paraffin sections $(7 \mu \mathrm{m})$ that were immunofluorescently stained using IBA1 antibodies. Cells were counted around the sagittal midline or coronal plane at the height of different brain areas. Within one plane (20 x magnification), only IBA1positive cells that 1) fully colocalized with DAPI-positive nuclei, and 2) had a clear cell soma and at least two clear protrusions were counted in the different regions of the brain. Microglia number per frame was corrected for surface area. $M f p 2^{-/-}$and control mice of different ages were used ( $\left.n=4-5 / g r o u p\right)$.

Fluorescence intensity quantification. IBA1 immunofluorescence intensity was measured in different brain regions such as brainstem, visual cortex and thalamus within one plane of $4 \mathrm{x}$ magnification. Using the Image J software (Fiji), channels were first split, background signal was subtracted by selecting an area of the section that expressed noise fluorescence, the area of interest was selected, and mean gray value was subsequently measured at the same area size per brain region.

\section{Real-time quantitative PCR (RT-qPCR)}

Total RNA was isolated from brain tissue using Trizol reagent (Invitrogen, California, USA). Subsequently, cDNA was generated from $1 \mu \mathrm{g}$ RNA using the QuantiTect Reverse Transcription Kit (QIAGEN, Venlo, The Netherlands). Real time PCR was performed as previously described [33] using an ABI PRISM 7500 Real Time PCR instrument (Applied Biosystems, Lennik, Belgium). Primers and probes were either designed using Primer Express Software (Applied Biosystems) or ordered from Applied Biosystems as premade Taqman Gene Expression assays (Il1b, Mm011336189_m1; Tnfa, Mm.PT.58.12575861; Cx3cr1, Mm0262011_s1; Tlr2, Mm.PT.58.45820113; Tspo, Mm.PT.58.43313736; F4/80 or Emr1, Mm.PT.58.11087779; Cholesterol 25-hydroxylase (25OHChol), Mm.PT.58.42792394). Mouse Aif primer (Mm00479862_g1) was ordered from Life Technologies (Gent, Belgium). Assays were performed in duplicate or triplicate in $10 \mu \mathrm{L}$ TaqMan Fast Universal PCR Master Mix (Applied Biosystems). Relative expression levels of the target genes were calculated taking into account the amplification efficiency as described [34]. The relative expression levels of the target genes were calculated as a ratio to the housekeeping gene $\beta$-actin. 


\section{Statistical analyses}

All data except some behavioral tests were analyzed with GraphPad Prism software (version 5.0 and 6.0, San Diego, CA). Statistical analyses were carried out using unpaired and paired, two-sided Student's $t$-test, one-way ANOVA, two-way ANOVA or two-way repeated measures (RM) ANOVA followed by the Bonferroni post hoc test. Data are shown as mean \pm Standard Error of the Mean (SEM) and statistical significance was set at $p<0.05$. SPSS Statistics software was used for three-way ANOVA and three-way RM ANOVA

BAEP test. Three-way RM ANOVA with genotype, age, and peak as sources of variation and two-way RM ANOVA per age with genotype and peak as sources of variation were used to evaluate disease progression of $\mathrm{Mfp}^{--/}$mice. Two-way ANOVA with age and model as independent variables was used for evaluation of interpeak latencies.

$O F$ and PA test. Two-way ANOVA with genotype and age as sources of variation was used to evaluate disease progression of $\mathrm{Mfp}^{-/-}$mice. Subsequently, performance of $M f p 2^{-/}$mice (expressed relative to their respective wildtype values) was directly compared using two-way ANOVA with age and model as independent variables.

The Holm-Sidak and Bonferroni method was used for multiple comparisons. Independent samples ttest was used to compare performance at pathological end stages. 


\section{RESULTS}

\section{Failure of anti-inflammatory drugs to suppress neuroinflammation}

In order to determine whether microgliosis contributes to CNS pathology in Mfp2 $2^{-/-}$mice, we first attempted to suppress neuroinflammation by administering anti-inflammatory drugs. Minocycline, previously shown to be neuroprotective through its anti-inflammatory activity in neurodegenerative disease mouse models $[14,15,30]$ was administered for 6 weeks. Brains of Mfp2 $^{-/}$and control mice were analyzed by IHC detecting the microglial marker IBA1. As previously shown, IBA1 immunoreactivity is increased in different CNS areas of $\mathrm{Mfp}^{-/-}$compared to control mice, consistent with development of extensive microgliosis. However, minocycline treatment was unable to reduce microgliosis in $\mathrm{Mfp}^{-/-}$mice (Fig. 1A). Also the PPARy agonist pioglitazone, known to ameliorate Parkinson's and Alzheimer's disease models by reducing inflammation [16, 17] was ineffective in decreasing IBA1 immunoreactivity (Fig. 1B). In addition, modified transcript levels of inflammatory markers Tnfa, Mpeg1, II6, II1b and Arg1 in Mfp2-/- brain [7] were unaltered after pioglitazone treatment (Data not shown). Finally, ADIOL shown to prevent experimental autoimmune encephalomyelitis by regulating inflammatory responses in microglia [18] did not change microglial reactivity in the Mfp $^{-/-}$ brain (not shown). During all anti-inflammatory treatments, clinical scores based on weight, gait, ataxia, alertness, cataract, and kyphosis were weekly measured, but no significant differences between treated and untreated mice in both genotypes were detected (shown for pioglitazone in Fig. 1C). Accordingly, treatments with these anti-inflammatory drugs were unable to ameliorate the severe neurological phenotype and clinical deterioration of $\mathrm{Mfp}^{-/-}$mice. Therefore, we searched for other strategies to eliminate microglia specifically.

\section{CSF1R inhibition depletes microglia}

Although we have previously documented microglial proliferation in MFP2 deficiency [7], the promitogenic factors involved were not defined. Microglia in the adult brain are dependent on CSF1R signaling for their survival. During neurodegeneration, it was shown that the ligands of CSF1R, namely CSF1 and especially IL-34, induce microglial proliferation [21, 25, 35]. Analysis of transcript levels in brain tissue demonstrated elevated expression of I/34 (7.7-fold) but not of Csf1 in Mfp2 $2^{-/-}$mice compared to control mice at 3 months of age (Fig.2A,B).

Given the increased II34-CSF1R signaling in Mfp2 ${ }^{-/-}$brain, we attempted to eliminate microglia by administering PLX5622, an orally bioavailable selective CSF1R inhibitor that crosses the blood-brain barrier [22, 36, 37]. PLX5622 was mixed into standard rodent chow at 1200 ppm and administered to 6-weeks-old $\mathrm{Mfp}^{-/-}$and control mice for a period of 10 weeks. PLX treatment depleted nearly all IBA ${ }^{+}$ microglia in control brain (shown for brainstem in Fig. 3A,B; for visual cortex at a higher magnification in Fig. 3H,I) and markedly diminished the microglial response in Mfp2 ${ }^{-/-}$brain (shown for brainstem in Fig. 3C,D and visual cortex Fig. 3J,K). In the latter, the remaining microglia exhibited an enlarged morphology with thickened protrusions (Fig. 3K). Quantification of IBA1 fluorescence in different brain regions of control mice verified an $80 \%, 94 \%$ and $93 \%$ reduction in brainstem, visual cortex and thalamus, respectively (Fig. 3E-G). In Mfp2\%- brain, the PLX5622 reduced IBA1 fluorescence by $66 \%$, 
$72 \%$ and $57 \%$ in brainstem, visual cortex and thalamus, respectively (Fig. 3E-G). Quantification of IBA1+ cell numbers revealed a 95-98\% reduction in control brain, and a 70-73\% reduction in $M f p 2 \%$ brain (Fig. $3 \mathrm{~L}, \mathrm{M}$, shown for brainstem and visual cortex). In summary, PLX treatment significantly reduced, but could not irradicate microglia in Mfp2-- mice, suggesting that CSF1R signaling is important for the robust microgliosis. The residual microglia in PLX-treated $\mathrm{Mfp}^{-/-}$mice still display a reactive morphology.

\section{CSF1R inhibition diminishes expression of microglial activation markers, but does not affect astrogliosis}

To further define microglial features, transcript levels of microglia-related genes were analyzed in brainstem of $\mathrm{Mfp}^{--}$and control mice treated with PLX or vehicle. Transcripts of the general marker Iba1, the homeostatic marker $C \times 3 c r 1$ and the activation marker $F 4 / 80$ were significantly increased in Mfp2-- mice as compared to control mice. After PLX treatment, microglial transcript levels were reduced by $80 \%$ (Iba1), $82 \%$ ( $C \times 3 \mathrm{cr} 1$ ), and $71 \%$ ( $F 4 / 80)$ in control brainstem whereas this reduction was less pronounced in $\mathrm{Mfp}^{-/}$brainstem being 44\% (Iba1), 57\% (Cx3cr1), and 32\% (F4/80) (Fig. 4A-C).

Apart from reduced gene expression after PLX treatment, we determined whether $F 4 / 80$ activation was also reduced at the protein level by $\mathrm{IHC}$ analysis. $\mathrm{F} 4 / 80^{+}$cells are not detectable in control brain (not shown), but we found a significant reduction of F4/80 activation in the entire $M$ fp $^{-/}$brain after PLX treatment (Fig. 4D,E). However, F4/80+ cells were not completely eliminated (Fig.4E), which confirms that PLX treatment could only diminish the strongly activated phenotype of $\mathrm{Mfp}^{-1-}$ microglia.

Microgliosis is associated with astrogliosis in CNS pathologies and it is hypothesized that immunologically activated microglia initiate and maintain astrogliosis [38]. To determine whether PLX treatment impacts on astrogliosis in $\mathrm{Mfp}^{-/}$brain [9], IHC for the astroglial marker GFAP was performed. We found increased immunoreactivity throughout the $M f p 2^{-/}$brain as compared to controls (not shown), but PLX treatment did not affect number nor shape of $\mathrm{GFAP}^{+}$cells in any brain region (Fig. 4F,G). Taken together, the results demonstrate that PLX treatment specifically downregulates but does not deplete microglial markers whereas astroglial markers remain unaffected in $\mathrm{Mfp}^{-1-}$ mice.

\section{CSF1R inhibition cannot reverse the inflammatory response in the brain}

To explore how microglial depletion affects the inflammatory state of the brain, several immune-related genes were analyzed in $\mathrm{Mfp}^{--}$and control brainstem after PLX or vehicle treatment. Transcript levels of pro-inflammatory markers Tnfa, $I I 1 \mathrm{~b}, \mathrm{Tl} 2 \mathrm{2}$ and $25 \mathrm{OHChol}$, and the general inflammatory marker Tspo were significantly increased in vehicle-treated $M f p 2^{-/}$relative to control brain (Fig. 5A-E). After PLX treatment, transcript levels of Tnfa and $I I 1 b$ were significantly downregulated in control brain. Surprisingly, none of the transcripts were significantly changed in PLX-treated versus vehicle-treated Mfp2-/ mice (Fig. 5A-E). Thus, despite the substantial reduction in numbers of microglia, CSF1R inhibition cannot prevent the development of an inflamed brain.

\section{Depletion of microglia does not improve clinical score and grip strength}


Mice were clinically evaluated on a weekly base by measurements of weight, grip strength, and clinical score to identify whether microglial diminution impacts the clinical phenotype and performances of Mfp2- mice. Clinical scores were given based on general activity, gait, ataxia, kyphosis, and cataract. Mfp2-

1- mice show an early-onset decreased weight [9, 29], but there were no significant differences observed between PLX-and vehicle-treated animals (Fig. 6A). Worsening of clinical scores with age ran parallel between PLX- and vehicle-treated $M f p 2^{-/-}$mice (Fig. 6B). In addition, PLX treatment did not improve decreased grip strength of both front paws (Fig. 6C) and all paws together (Fig. 6D). We previously showed that $\mathrm{Mfp}^{-/}$mice are not able to remain hanging on an inverted grid (Beckers and Baes, unpublished observations). Comparison of PLX-treated with vehicle-treated mice revealed that PLXtreated $M f 2^{--}$mice similarly fail to retain on the inverted grid (Fig. 6E). Based on these results, we conclude that CSF1R inhibition and subsequent reduced microglial numbers did not halt nor delay clinical deterioration of $\mathrm{Mfp}^{-/-}$mice.

\section{Microglial depletion did not prevent behavioral and cognitive impairment}

Given the result that significant reduction of microglia did not reduce the inflammatory state of the brain nor improve the clinical phenotype of $\mathrm{Mfp}^{-/-}$mice, we sought to determine whether PLX treatment could prevent behavioral anomalies. Therefore, prior to sacrifice at 16 weeks of age, fear conditioning as an index of learning ability was assessed by means of the passive avoidance (PA) test. The test phase is preceded by a training phase during which mice received punishment to learn to avoid an aversive context. Training phase was not shown as there were no significant differences between genotypes and treatments. During the test phase, Mfp2 ${ }^{-/}$mice treated with PLX stepped as quickly into the room delivering the electroshock compared to vehicle treated mutants whereas the control mice had learned to avoid the shock, indicating that PLX treatment could not prevent the cognitive decline (Fig. 7A). Notably, PLX-treated control mice performed significantly better in the PA test compared to vehicletreated control mice (Fig. 7A).

Locomotor activity and explorative behavior were assessed by video-tracking mice in an open field (OF) environment. There was no change in corner entries (Fig. 7B) in PLX-versus vehicle-treated mice which shows that PLX treatment could not improve locomotor activity in $\mathrm{Mfp}^{--}$mice. We previously defined that $\mathrm{Mfp}^{--}$- mice spent increased time in the center and showed a decreased average distance to center as indications of disturbed explorative behavior (Beckers and Baes unpublished data). PLX treatment could not avoid disturbed explorative behavior in $\mathrm{Mfp}^{-/}$mice shown by similarly increased time in center (Fig.7C) and decreased distance to center (Fig. 7D) compared to vehicle-treated mice.

In summary, microglial diminution by PLX treatment in $\mathrm{Mfp}^{-/-}$mice was not able to prevent disturbed locomotion, exploration and cognition.

\section{Depletion of microglia did not prevent dysfunctional neuronal transmission}

BAEP recordings revealed previously that $\mathrm{Mfp}^{-/-}$mice exhibit delayed neuronal transmission and decreased amplitudes of the auditory signal (Beckers and Baes, unpublished data). As severity of neuronal dysfunction is correlated with the extent of microgliosis, we wanted to discern whether CSF1R 
inhibition improves neuronal functioning in the $M f 2^{---}$brain. Therefore, auditory brainstem responses were conducted in $\mathrm{Mfp}^{-/}$and control mice after 10 weeks of PLX or vehicle treatment. We found that mean peak latencies were not changed by PLX treatment compared to vehicle treatment in both control (Fig. 8A) and $\mathrm{Mfp}^{-/}$(Fig. 8B) mice. This was confirmed by direct comparison of PLX-treated $\mathrm{Mfp}^{-/}$ with PLX-treated control mice that reveals significant delayed peak responses in PLX-treated Mfp2-versus control mice (Fig. 8C), consistent with our previous non-treatment data (Beckers and Baes, unpublished). Furthermore, PLX treatment did not affect the reduced amplitudes in Mfp2--mice (Fig. 8D). 


\section{DISCUSSION}

The key feature of pathology in Mfp2 ${ }^{-/}$mice is the development of strong and progressive microgliosis. The consequences and impact of this innate immune response on neuronal functioning and the clinical phenotype is still unknown. Here, we attempted to block neuroinflammation by administering antiinflammatory agents or the selective CSF1R inhibitor PLX5622.

Whereas the anti-inflammatory drugs minocycline, pioglitazone and ADIOL were unable to prevent the development of microgliosis, PLX5622 effectively reduced microglial numbers. Microglia were completely eliminated from control (>95\%), but incompletely $(-70 \%)$ from $M f p 2^{-/}$brain such that numbers of surviving microglia in $\mathrm{Mfp}^{-/-}$mice remain higher than in vehicle-treated control mice. Based on transcript levels, we showed that the driver for CSF1R activation and microglial proliferation is IL-34 but not CSF1. The incomplete microglial elimination from $M f 2^{-/-}$brain indicates that PLX5622, at this concentration, cannot fully block the activation of CSF1R by the strongly upregulated IL-34 [23, 25]. Alternatively, it is plausible that other pro-mitogenic regulators induce microgliosis in $\mathrm{Mfp}^{-1-}$ brain. TREM2, a cell surface receptor of the Ig-superfamily, which senses a broad class of anionic lipids and acts in cooperation with CSF1R to sustain microglial survival, seems to be a good candidate given the involvement of MFP2 in lipid metabolism [39]. It would therefore be interesting to explore whether TREM2 signaling is activated. It should also be noted that CSF1R blockers are well established to eradicate microglia in physiological conditions $[21,22,25]$ but that they have been less applied in chronic neuroinflammatory conditions. In models of Alzheimer Disease (AD), PLX agonists were shown to significantly reduce microglial numbers but contradictory data were obtained whether plaque associated or non-associated microglia were preferentially depleted [24, 26]. In any case, $20-50 \%$ microglia remained depending on the dose used, which is consistent with our findings.

After PLX treatment, the remaining microglia in $\mathrm{Mfp}^{-/-}$brain still adopt an inflammatory activated morphology. This was not surprising, given that the factors originating from neurons causing the microglial response are unaltered. More unexpectedly, analysis of transcript levels of several inflammatory markers in brain revealed that despite strong reduction of microglial numbers, the inflammatory responses were not substantially changed by CSF1R inhibition. Because the PLX treatment did neither affect number nor shape of astrocytes, we assume that the residual reactive microglia are the source of the unaltered inflammatory burden. We can exclude that this is triggered by systemic inflammation as inflammatory markers are unchanged in the circulation and in peripheral organs and there is no influx of monocytes into the $M f p 2^{-/-}$brain $([7,10]$ and unpublished data). In order to compensate for the loss of most microglia in PLX-treated Mfp $^{-/-}$brain, the remaining microglia might produce higher levels of inflammatory markers in response to the unaltered but affected brain environment. In this respect, it would be interesting to analyze the remaining microglia by single cell sequencing approaches. Our findings diverge from those in PLX-treated AD models in which a substantial reduction in expression of some but not all inflammatory genes was observed in parallel with reduced microglial numbers $[24,26]$. Also in prion-diseased mice the CSF1R inhibitor GW2580 significantly reduced microglial proliferation as well as expression of pro-inflammatory cytokines [25]. It 
should however be noted that levels of inflammatory markers in untreated $M f p 2^{-/}$brain are much more elevated than in the neurodegenerative disease models. This difference in microglial phenotype might explain the inefficient decrease of pro-inflammatory markers.

The role of microglia in learning and memory in physiological conditions is still controversial. Several reports demonstrated profound defects in learning and memory processes in genetically modified mouse models that have dramatically reduced numbers of microglia $[40,41]$. On the contrary, it was shown that short- and long-term microglial depletion by CSF1R inhibition does not impair explorative behavior, locomotion and anxiety in an OF environment, cognition by both contextual fear-conditioning and by the Barnes maze test, and locomotion on an accelerating rotarod [21]. Learning was even enhanced in mice with chronic depletion of microglia [21]. Also in our study, microglia-depleted control mice were able to learn the PA task significantly better than microglia-intact mice.

The PLX treatment did however not improve any of the clinical parameters, neuronal dysfunction or behavioral deficits of MFP2-deficient mice. This contrasts with the previously mentioned studies of microglia depletion in neurodegenerative conditions in which the reduced inflammatory profile correlated with neurological improvement, enhanced memory or slower disease progression. Several hypotheses can be proposed to explain the failure to circumvent the clinical deterioration in $\mathrm{Mfp}^{-/}$mice: (1) Although microglial cell numbers were significantly decreased by CSF1R inhibition, the inflammatory activated state of the brain could not be suppressed. Consistent with this hypothesis, the remaining microglia in PLX-treated Mfp2 ${ }^{-/}$mice still adopt a strongly activated morphology. (2) Mfp2 $2^{-/}$microglia do not contribute to clinical deterioration and neuronal dysfunction of $\mathrm{Mfp}^{-/}$mice, suggesting that neuropathology in $\mathrm{Mfp}^{-/-}$mice is mainly caused by neuronal deficits that cannot be suppressed by antiinflammatory treatments. The latter is unlikely as we previously found that neuronal dysfunction, locomotor activity, and explorative behavior are more severe in constitutive $\mathrm{Mfp}^{-/}$mice compared to mice with neural-specific loss of MFP2. Furthermore, we showed that the severity of clinical and neuronal impairments positively correlated with stronger microgliosis and a distinct aberrant microglia phenotype (Beckers and Baes, unpublished). (3) Other cells such as astrocytes and oligodendrocytes might participate in the development of neuropathology in $\mathrm{Mfp}^{-/}$mice. However, various reasons refute this hypothesis. First, astrogliosis is less pronounced compared to microgliosis in our disease model [9]. Second, no myelin abnormalities were found in $M f p 2^{--/}$mice which suggests that oligodendroglia work properly [10].

Together, the inefficacy of anti-inflammatory agents to reduce microgliosis and the persistent inflammatory milieu despite reduced numbers of microglia after PLX treatment strongly indicates that the mechanisms governing neuroinflammation in MFP2 deficiency diverge from those in a neurodegenerative environment. It is plausible that this unstoppable inflammatory response is related to a unique microglial phenotype induced by the loss of MFP2 from these immune cells. A particular hallmark of this state is that it is accompanied by impaired neurological function but not with neuronal loss. It would therefore be worthwhile to further investigate the role of peroxisomal $\beta$-oxidation in 
microglia by generating mice with microglia-selective deletion of MFP2. In summary, the strongly reduced numbers of reactive microglia but unchanged inflammatory environment in PLX5622 treated $M f p 2^{-1-}$ mice encumbers the outset of our studies to define the impact of microgliosis on their impaired neurological abilities. Still, the present findings are in agreement with our hypothesis that chronic neuroinflammation contributes to the neurological deterioration of these mice.

\section{ACKNOWLEDGMENTS}

The authors wish to thank Benno Das and Lies Pauwels for excellent technical assistance, Leen Van Aerschot for her help regarding mice transfer at the animal facility of Biological Psychology. This work was funded by grants from Fonds Wetenschappelijk Onderzoek Vlaanderen (G.0675.12 and G.0A15.13), and KU Leuven (OT12/78). 


\section{FIGURE LEGENDS}

Figure 1: Anti-inflammatory treatment does not suppress microgliosis nor prevent clinical deterioration in $\mathbf{M f p 2}^{-/-}$mice. (A) IBA1 staining of microglia (green) in inferior colliculus of $\mathrm{Mfp}^{-/-}$and control mice after 6 weeks of treatment with minocycline or vehicle. Representative picture $(n=4-5)(B)$ IBA1 staining of microglia (green) in brainstem of $\mathrm{Mfp}^{-/-}$and control mice after 6 weeks of treatment with pioglitazone or vehicle. Representative picture $(n=3)$. (C) Clinical deterioration of $\mathrm{Mfp}^{-1}$ mice, represented by an increasing clinical score, is not restrained by pioglitazone treatment. Data of control mice were not shown as clinical scores were 0 for all mice at all ages. Error bars indicate SEM. $n=6$ 8/group.

Figure 2: Modified CSF1R signaling in $\mathbf{M f p 2}^{-{ }^{-}}$mice. (A,B) Transcript levels of the Csf1 ligand (A) are not significantly changed whereas transcripts of the II34 ligand $(B)$ are significantly induced in Mfp2-Imice. Transcript levels were analyzed in the brainstem of $\mathrm{Mfp}^{-1-}$ and control mice at 3 months of age. ${ }^{\star}$ Control versus Mfp2 ${ }^{-1}:{ }^{* \star \star} p<0.001$, ns $=$ not significant. Error bars indicate SEM. $n=4 /$ group.

Figure 3: Incomplete suppression of microgliosis in Mfp2 $^{-/-}$mice. 16-weeks-old Mfp2 $^{-/-}$and control mice were analyzed after a 10-week PLX or vehicle treatment. (A-D) IHC overview pictures of Iba1+ microglial cells in the brainstem of PLX- or vehicle-treated control and Mfp2-- mice. (E-G) Quantification of Iba1+ fluorescence in total brainstem (E), visual cortex (F) and thalamus (G) shows a $80-94 \%$ reduction in control mice and a 57-72\% reduction in $\mathrm{Mfp}^{-/-}$mice. $(\mathrm{H}-\mathrm{K}) \mathrm{IHC}$ pictures at a higher magnification (20X) of Iba1+ microglial cells in the visual cortex of PLX- or vehicle-treated control and Mfp2 $2^{-/}$mice. (L,M) Quantification of Iba1+ microglial cells in total brainstem (L) and total visual cortex (M) shows a 95-98\% reduction in control mice and a 70-73\% reduction in $M f p 2^{-/-}$mice. *Control versus

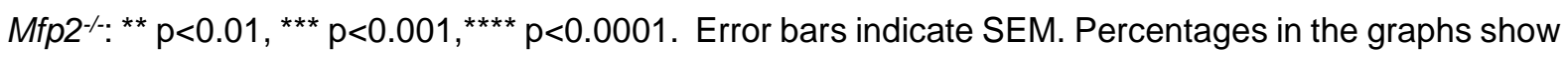
the reduction of microgliosis after PLX relative to vehicle treatment. $n=4-5 / g r o u p$.

Figure 4: Reduction of microglial but not astroglial activation in $\mathrm{Mfp2}^{--}$mice after PLX treatment. 16-weeks-old $\mathrm{Mfp}^{-/}$and control mice were analyzed after a 10-week PLX or vehicle treatment. (A) After PLX treatment, transcript levels of microglial markers are more reduced in control than in $M f p 2^{--}$ brainstem. Percentages in the graphs show the reduction of microglial transcripts after PLX relative to vehicle treatment. $(D, E)$ Reduction of F4/80+ microglial activation in visual cortex of PLX-treated Mfp2/- mice (E) versus vehicle-treated $M f p 2^{-/}$mice $(D) .(F, G)$ Astrogliosis is not significantly changed between vehicle- $(F)$ and PLX-treated $(G) M f p 2^{--/}$mice. Representative pictures are shown. ${ }^{*}$ Control versus Mfp $^{-1}$ : ${ }^{*} p<0.05,{ }^{\star \star \star} p<0.01,{ }^{\star \star *} p<0.001$. Error bars indicate SEM. $n=4$-5/group.

Figure 5: PLX5622 cannot reverse the inflammatory response in Mfp $^{-\%}$ CNS. Transcript level analysis of inflammatory markers in brainstem of 16-weeks-old $M f p 2^{-/}$and control mice were analyzed after a 10-week PLX or vehicle treatment. (A-B) After PLX treatment, transcript levels of Tnfa (A) and III (B) are significantly reduced in control brain but not in Mfp2 $2^{-/}$brain. (C-E) Transcript levels of 
neuroinflammatory markers TIr2, Tspo, and $25 \mathrm{OHChol}$ are not altered in both genotypes after PLX treatment. ${ }^{*}$ Control versus $\mathrm{Mfp}^{-/}{ }^{*}{ }^{*} \mathrm{p}<0.05$, ns $=$ not significant. Error bars indicate SEM. $n=5 /$ group.

Figure 6: CSF1R inhibition does not improve clinical parameters. (A) Reduced weights of $M f p 2^{-/}$mice as compared to control mice are not changed by PLX treatment. (B) Clinical deterioration of $\mathrm{Mfp}^{--/}$mice, represented by an increasing clinical score, is not improved by PLX treatment. (C,D) Decreased grip strength in front paws (C) and all paws together (D) in $M f p 2^{-/-}$mice is not improved by PLX treatment. Grip strengths were normalized to vehicle-treated control mice. (E) Both vehicle- and PLX-treated $M f p 2^{-/-}$mice fail to remain hanging on an inverted grid in contrast to efficient performances of control mice. Error bars indicate SEM. $n=9 / g r o u p$. Veh $=$ Vehicle.

Figure 7: PLX treatment cannot prevent cognitive and behavioral impairment. (A) PLX treatment improved fear-conditioned learning in control mice, but did not affect the severely impaired learning in $M f p 2^{-/}$mice. (B-D) Explorative locomotion in an open-field environment was assessed in vehicle- and PLX-treated $\mathrm{Mfp}^{-{ }^{-}}$and control mice after a 10-week treatment. PLX treatment did not influence number of corner entries (B), time in center (C) and mean distance to center (D) in both genotypes. ${ }^{*}$ Control versus Mfp $2^{-1 /}:{ }^{*} p<0.05,{ }^{* \star} p<0.01,{ }^{* \star *} p<0.001$, ns $=$ not significant. Error bars indicate SEM. $n=9 /$ group.

Figure 8: PLX treatment does not improve affected neuronal transmission. BAEP were analyzed in the brain of vehicle- and PLX-treated $M f p 2^{--}$and control mice after a 10-week treatment. (A-B) Brainstem responses were not altered in control (A) and $M f p 2^{--~}$ mice (B) after PLX treatment. (C) Comparison of PLX-treated Mfp $2^{-/-}$with PLXtreated control mice shows that neuronal transmission is significantly delayed in $\mathrm{Mfp}^{-{ }^{-/}}$mice, similar to vehicletreated $\mathrm{Mfp}^{-{ }^{--}}$mice. (D) Reduced amplitudes of brainstem responses in $M f p 2^{--\alpha}$ mice as compared to control mice are not improved by PLX treatment. ${ }^{*}$ Control versus $M f p 2^{--}:{ }^{* \star} p<0.01,{ }^{* \star \star} p<0.001, n s=$ not significant. Error bars indicate SEM. $n=$ 9/group. 


\section{REFERENCES}

1. Baes, M. and P. Aubourg, Peroxisomes, myelination, and axonal integrity in the CNS. Neuroscientist, 2009. 15(4): p. 367-79.

2. Baes, M. and P.P. Van Veldhoven, Mouse models for peroxisome biogenesis defects and beta-oxidation enzyme deficiencies. Biochim Biophys Acta, 2012. 1822(9): p. 1489-500.

3. Berger, J., et al., Peroxisomes in brain development and function. Biochim Biophys Acta, 2016. 1863(5): p. 934-55.

4. Kemp, S., J. Berger, and P. Aubourg, X-linked adrenoleukodystrophy: clinical, metabolic, genetic and pathophysiological aspects. Biochim Biophys Acta, 2012. 1822(9): p. 1465-74.

5. Cartier, N. and P. Aubourg, Hematopoietic stem cell transplantation and hematopoietic stem cell gene therapy in X-linked adrenoleukodystrophy. Brain Pathol, 2010. 20(4): p. 857-62.

6. Cartier, N., et al., Hematopoietic stem cell gene therapy with a lentiviral vector in $X$-linked adrenoleukodystrophy. Science, 2009. 326(5954): p. 818-23.

7. Verheijden, S., et al., Identification of a chronic non-neurodegenerative microglia activation state in a mouse model of peroxisomal beta-oxidation deficiency. Glia, 2015. 63(9): p. 160620.

8. Verheijden, S., et al., Central nervous system pathology in MFP2 deficiency: insights from general and conditional knockout mouse models. Biochimie, 2014. 98: p. 119-26.

9. Huyghe, S., et al., Peroxisomal multifunctional protein-2 deficiency causes motor deficits and glial lesions in the adult central nervous system. Am J Pathol, 2006. 168(4): p. 1321-34.

10. Verheijden, S., et al., Peroxisomal multifunctional protein-2 deficiency causes neuroinflammation and degeneration of Purkinje cells independent of very long chain fatty acid accumulation. Neurobiol Dis, 2013. 58: p. 258-69.

11. Ferdinandusse, S., et al., Clinical and biochemical spectrum of D-bifunctional protein deficiency. Ann Neurol, 2006. 59(1): p. 92-104.

12. Khan, A., et al., Neurodegeneration in D-bifunctional protein deficiency: diagnostic clues and natural history using serial magnetic resonance imaging. Neuroradiology, 2010. 52(12): p. 1163-6.

13. van der Knaap, M.S., et al., MRI as diagnostic tool in early-onset peroxisomal disorders. Neurology, 2012. 78(17): p. 1304-8.

14. Garrido-Mesa, N., A. Zarzuelo, and J. Galvez, Minocycline: far beyond an antibiotic. Br J Pharmacol, 2013. 169(2): p. 337-52.

15. Budni, J., et al., The Anti-Inflammatory Role of Minocycline in Alzheimer s Disease. Curr Alzheimer Res, 2016. 13(12): p. 1319-1329. 
16. Heneka, M.T., et al., Acute treatment with the PPARgamma agonist pioglitazone and ibuprofen reduces glial inflammation and Abeta1-42 levels in APPV717I transgenic mice. Brain, 2005. 128(Pt 6): p. 1442-53.

17. Pinto, M., et al., Pioglitazone ameliorates the phenotype of a novel Parkinson's disease mouse model by reducing neuroinflammation. Mol Neurodegener, 2016. 11: p. 25.

18. Saijo, K., et al., An ADIOL-ERbeta-CtBP transrepression pathway negatively regulates microglia-mediated inflammation. Cell, 2011. 145(4): p. 584-95.

19. Ginhoux, F., et al., Fate mapping analysis reveals that adult microglia derive from primitive macrophages. Science, 2010. 330(6005): p. 841-5.

20. Erblich, B., et al., Absence of colony stimulation factor-1 receptor results in loss of microglia, disrupted brain development and olfactory deficits. PLoS One, 2011. 6(10): p. e26317.

21. Elmore, M.R., et al., Colony-stimulating factor 1 receptor signaling is necessary for microglia viability, unmasking a microglia progenitor cell in the adult brain. Neuron, 2014. 82(2): p. 380-97.

22. Valdearcos, M., et al., Microglia dictate the impact of saturated fat consumption on hypothalamic inflammation and neuronal function. Cell Rep, 2014. 9(6): p. 2124-38.

23. Olmos-Alonso, A., et al., Pharmacological targeting of CSF1R inhibits microglial proliferation and prevents the progression of Alzheimer's-like pathology. Brain, 2016. 139(Pt 3): p. 891907.

24. Spangenberg, E.E., et al., Eliminating microglia in Alzheimer's mice prevents neuronal loss without modulating amyloid-beta pathology. Brain, 2016. 139(Pt 4): p. 1265-81.

25. Gomez-Nicola, D., et al., Regulation of microglial proliferation during chronic neurodegeneration. J Neurosci, 2013. 33(6): p. 2481-93.

26. Dagher, N.N., et al., Colony-stimulating factor 1 receptor inhibition prevents microglial plaque association and improves cognition in 3xTg-AD mice. J Neuroinflammation, 2015. 12: p. 139.

27. Feng, X., et al., Colony-stimulating factor 1 receptor blockade prevents fractionated wholebrain irradiation-induced memory deficits. J Neuroinflammation, 2016. 13(1): p. 215.

28. Acharya, M.M., et al., Elimination of microglia improves cognitive function following cranial irradiation. Sci Rep, 2016. 6: p. 31545.

29. Baes, M., et al., Inactivation of the peroxisomal multifunctional protein-2 in mice impedes the degradation of not only 2-methyl-branched fatty acids and bile acid intermediates but also of very long chain fatty acids. J Biol Chem, 2000. 275(21): p. 16329-36.

30. Fan, R., et al., Minocycline reduces microglial activation and improves behavioral deficits in a transgenic model of cerebral microvascular amyloid. J Neurosci, 2007. 27(12): p. 3057-63.

31. D'Hooge, R., et al., Neurocognitive and psychotiform behavioral alterations and enhanced hippocampal long-term potentiation in transgenic mice displaying neuropathological features of human alpha-mannosidosis. J Neurosci, 2005. 25(28): p. 6539-49. 
32. Hulshagen, L., et al., Absence of functional peroxisomes from mouse CNS causes dysmyelination and axon degeneration. J Neurosci, 2008. 28(15): p. 4015-27.

33. Bottelbergs, A., et al., Peroxisome deficiency but not the defect in ether lipid synthesis causes activation of the innate immune system and axonal loss in the central nervous system. J Neuroinflammation, 2012. 9: p. 61.

34. Giulietti, A., et al., An overview of real-time quantitative PCR: applications to quantify cytokine gene expression. Methods, 2001. 25(4): p. 386-401.

35. Zelante, T. and P. Ricciardi-Castagnoli, The yin-yang nature of CSF1R-binding cytokines. Nat Immunol, 2012. 13(8): p. 717-9.

36. Hamilton, J.A. and A. Achuthan, Colony stimulating factors and myeloid cell biology in health and disease. Trends Immunol, 2013. 34(2): p. 81-9.

37. Cavnar, M.J., et al., KIT oncogene inhibition drives intratumoral macrophage M2 polarization. J Exp Med, 2013. 210(13): p. 2873-86.

38. Zhang, D., et al., Astrogliosis in CNS pathologies: is there a role for microglia? Mol Neurobiol, 2010. 41(2-3): p. 232-41.

39. Wang, Y., et al., TREM2 lipid sensing sustains the microglial response in an Alzheimer's disease model. Cell, 2015. 160(6): p. 1061-71.

40. Rogers, J.T., et al., CX3CR1 deficiency leads to impairment of hippocampal cognitive function and synaptic plasticity. J Neurosci, 2011. 31(45): p. 16241-50.

41. Parkhurst, C.N., et al., Microglia promote learning-dependent synapse formation through brain-derived neurotrophic factor. Cell, 2013. 155(7): p. 1596-609. 
Figure 1
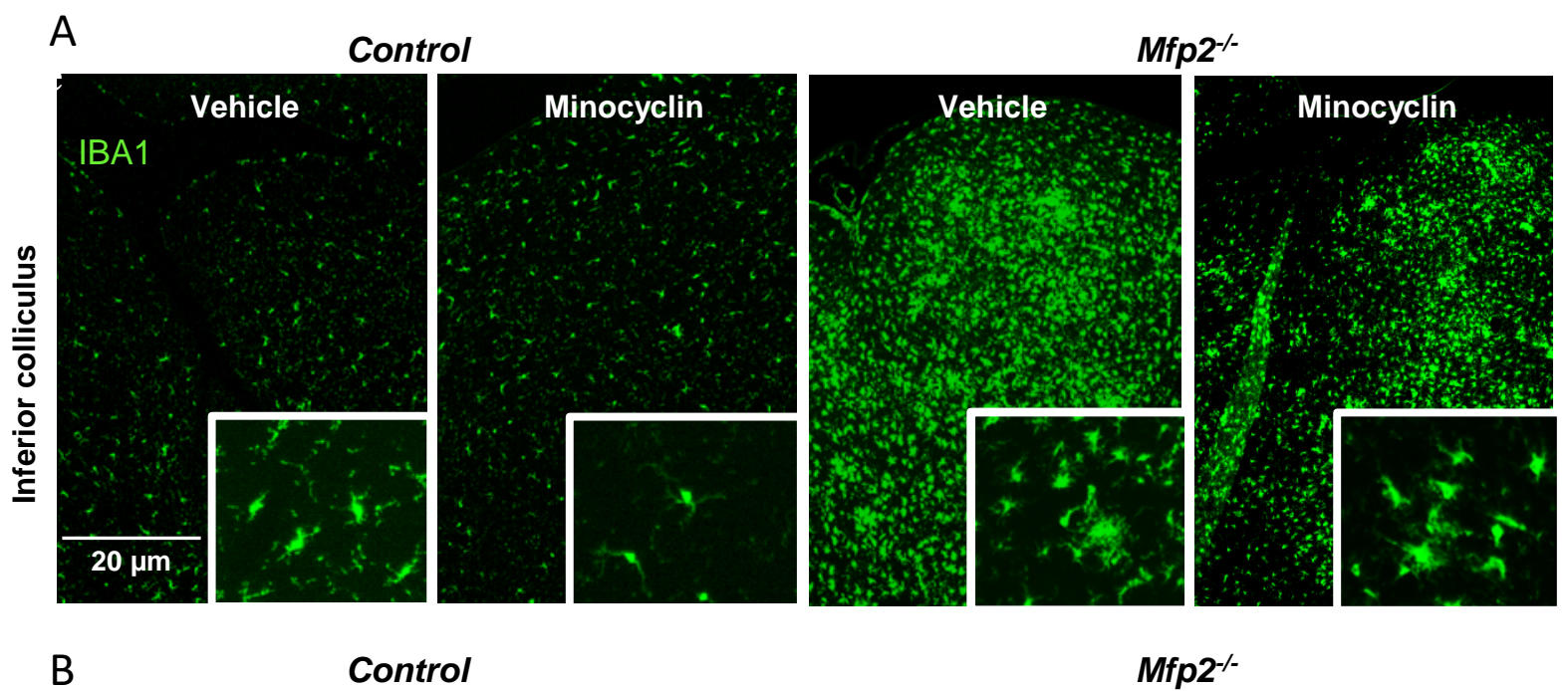

Mfp2---
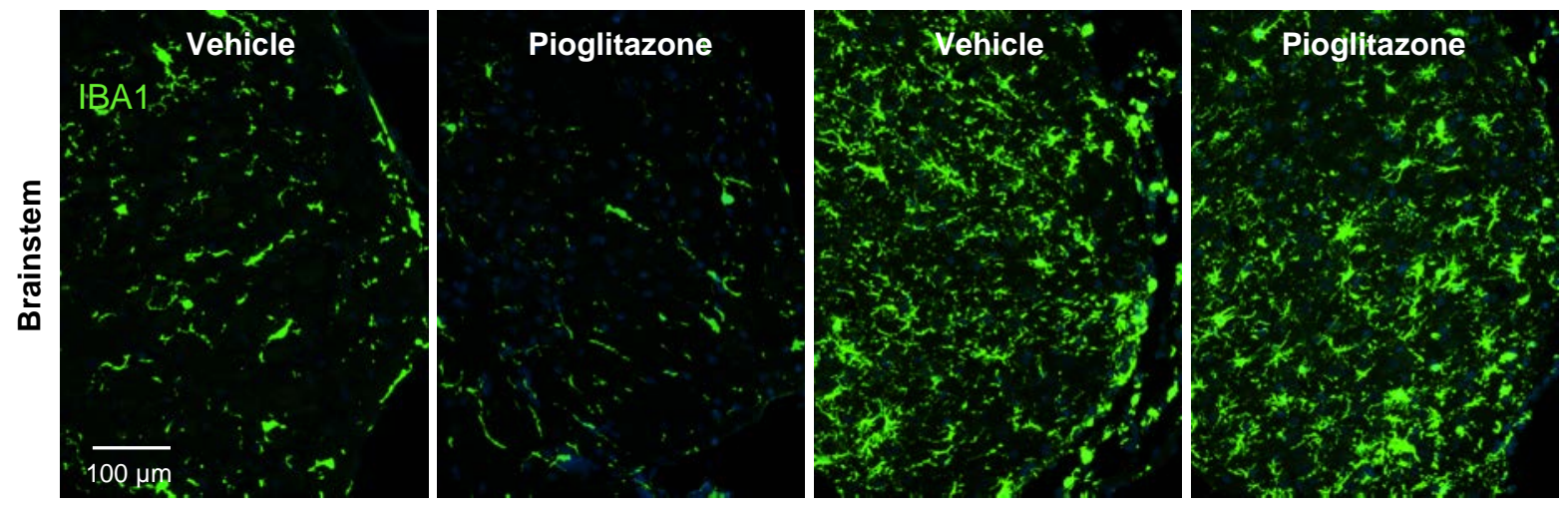

C

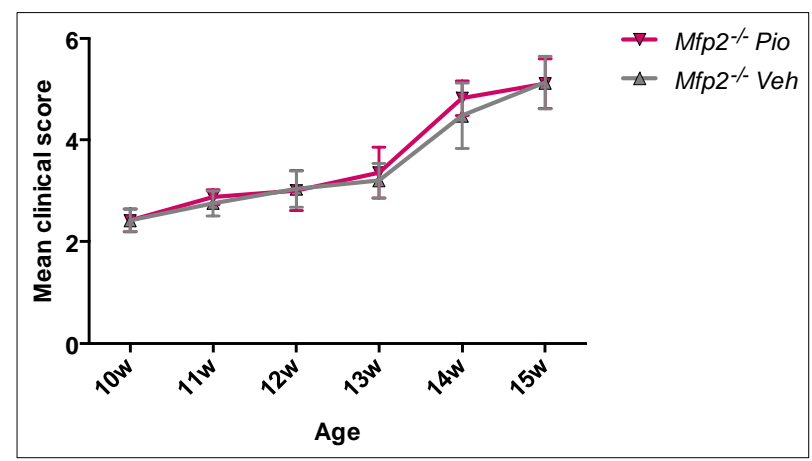


Figure 2

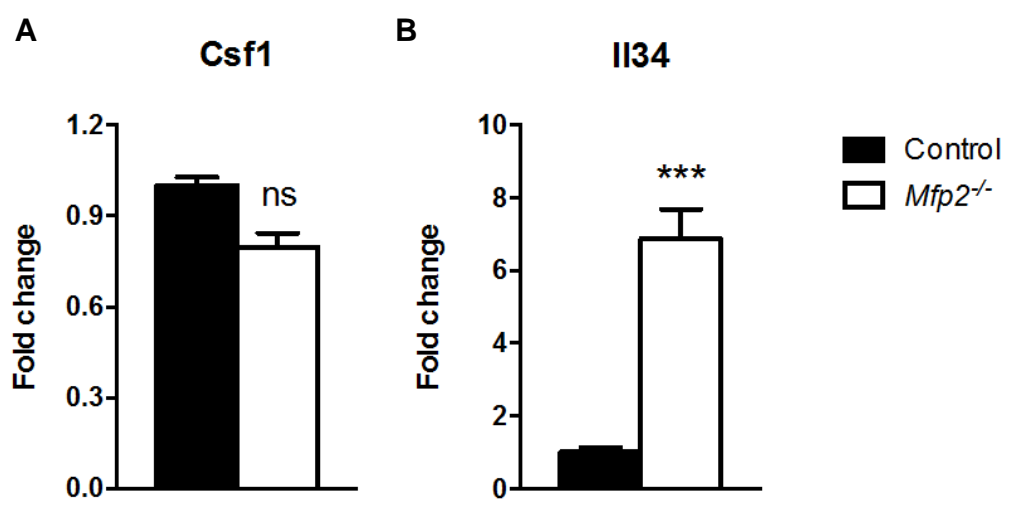


Figure 3

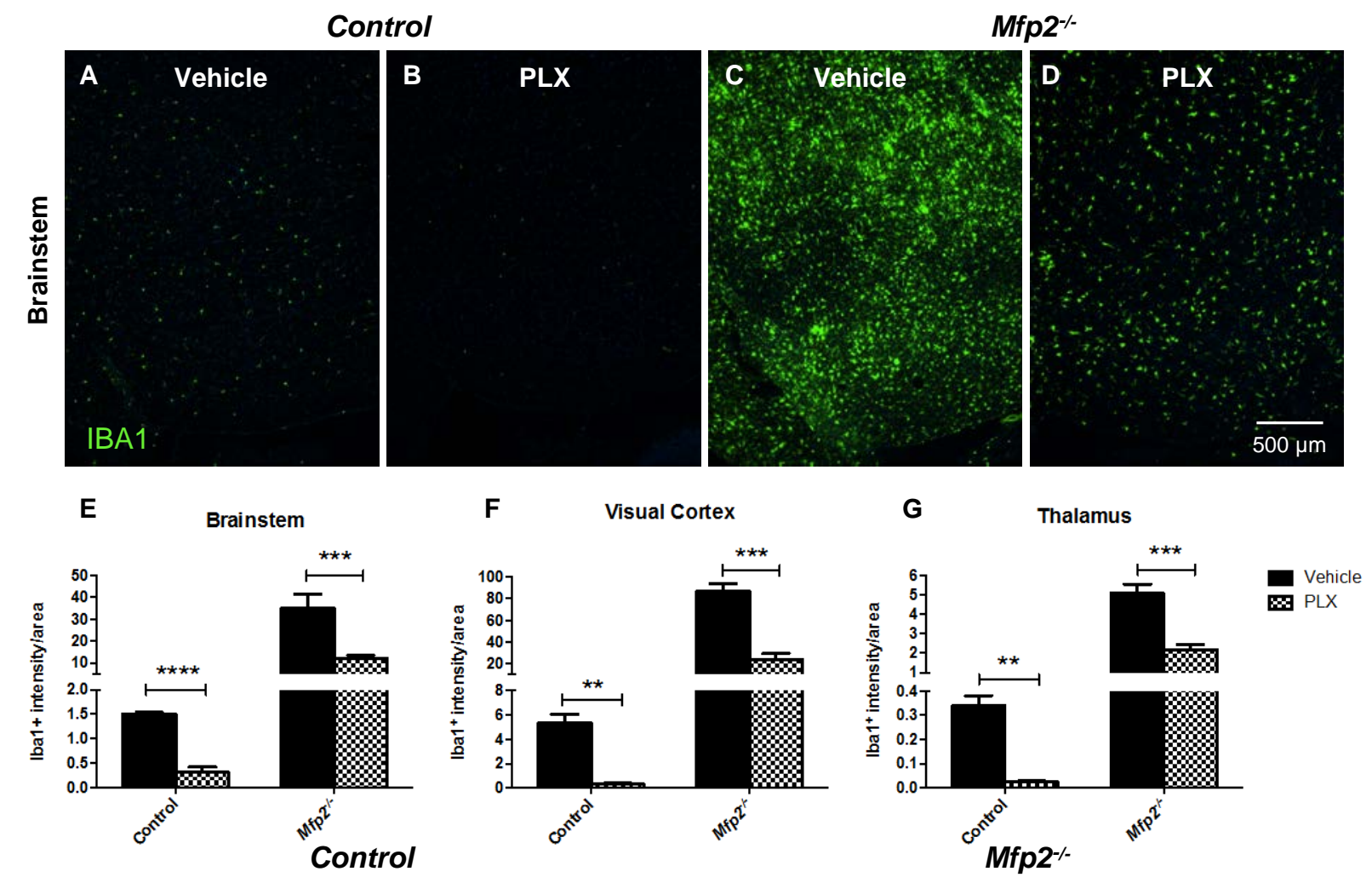

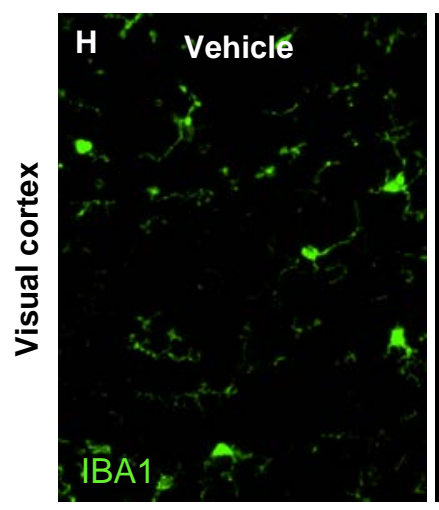

L

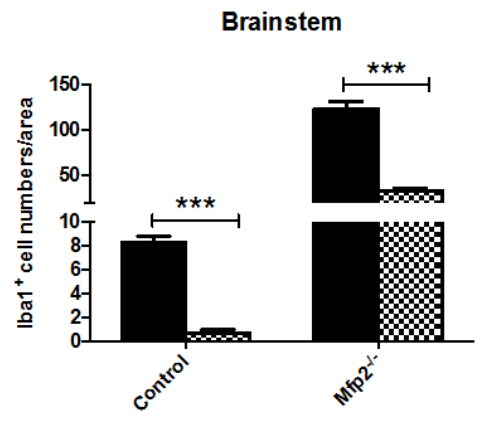

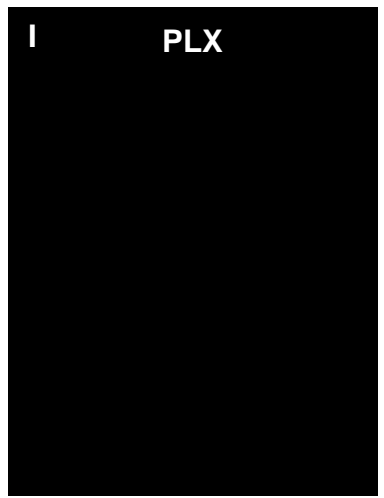
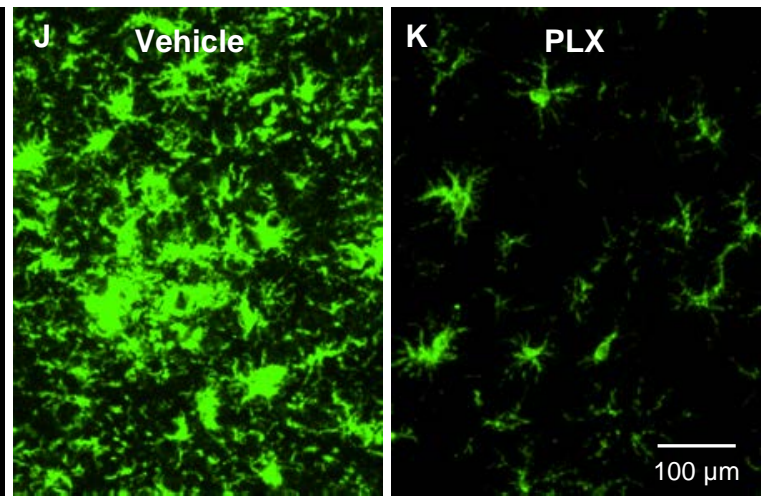

M

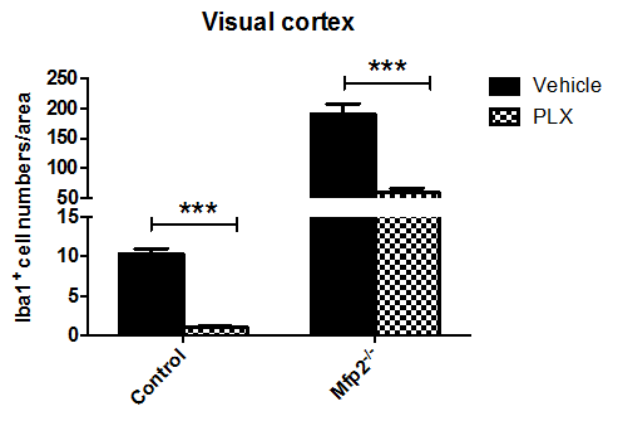


Figure 4
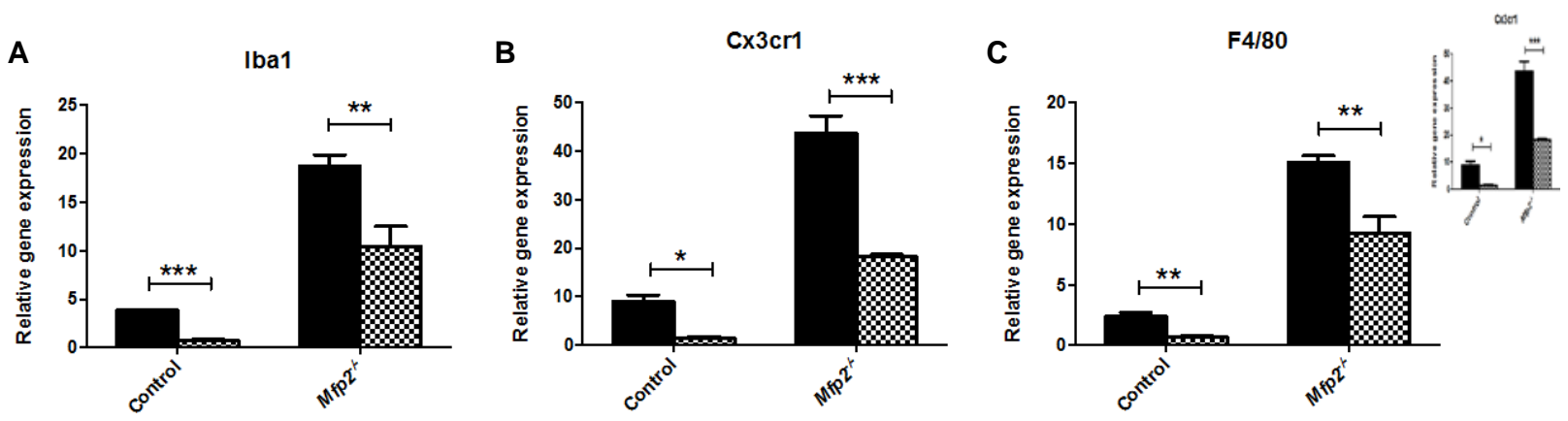

Mfp2-
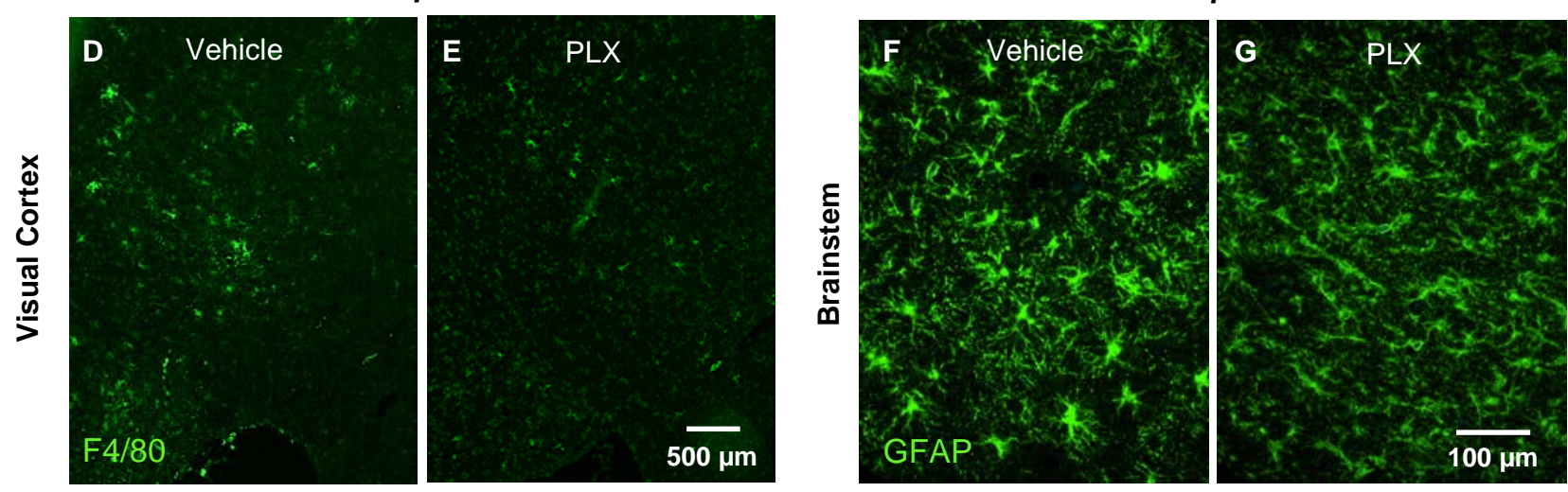
Figure 5
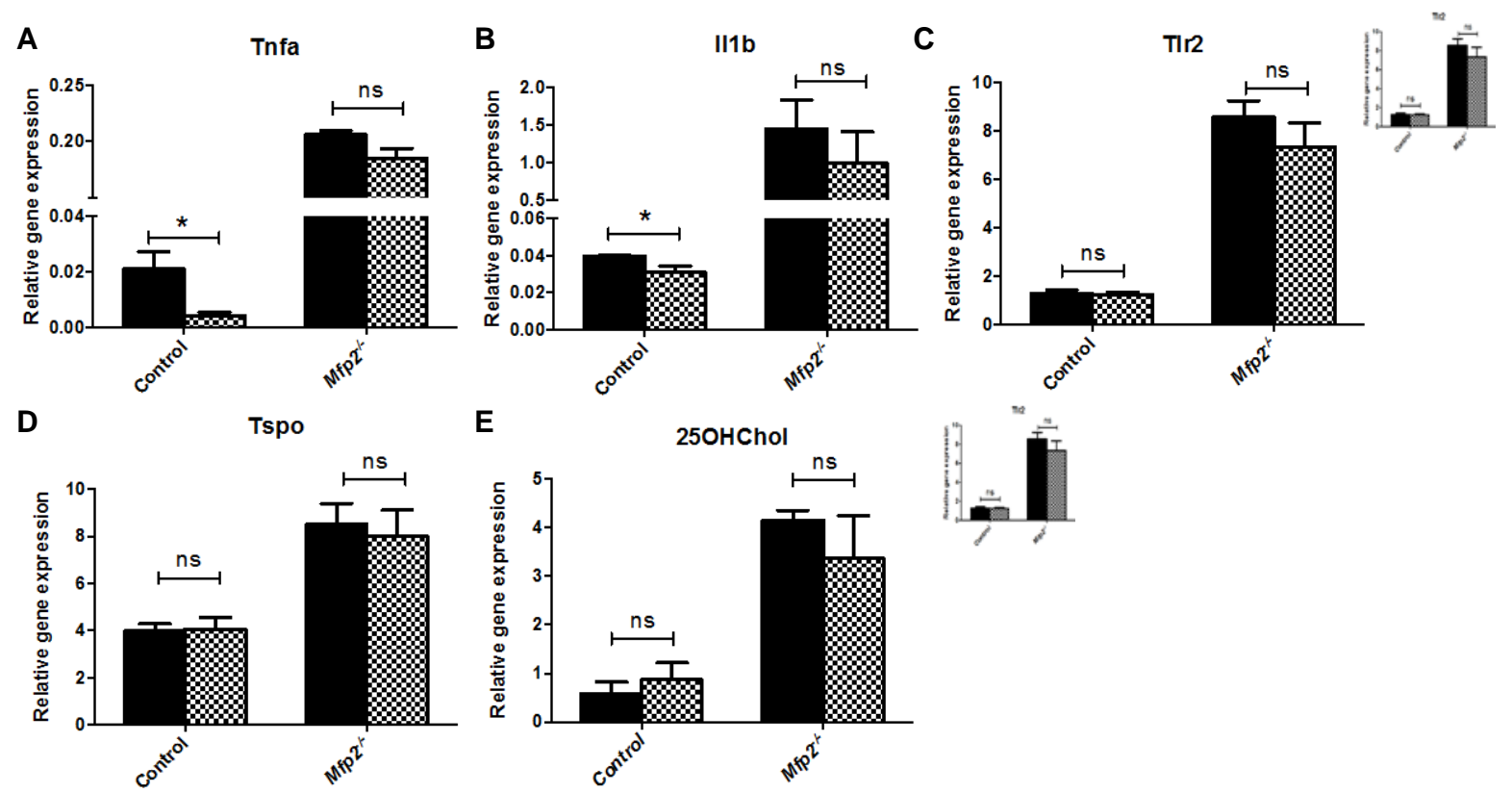
Figure 6

A

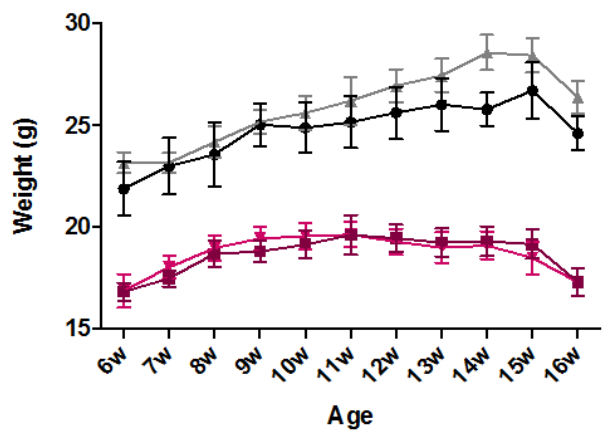

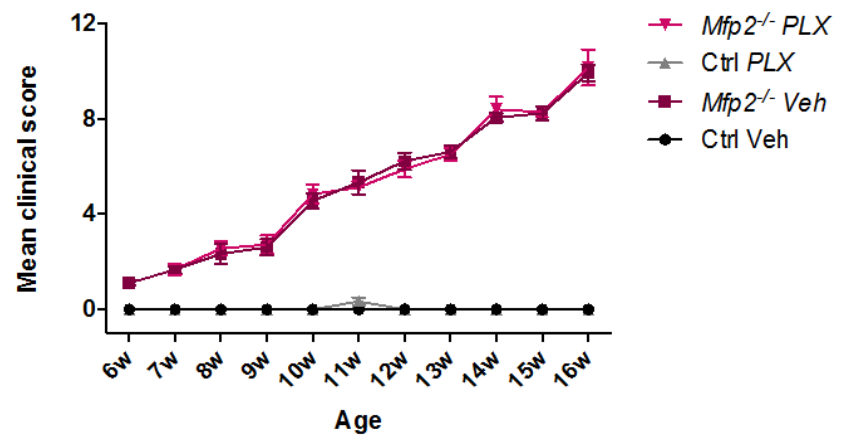

C

D

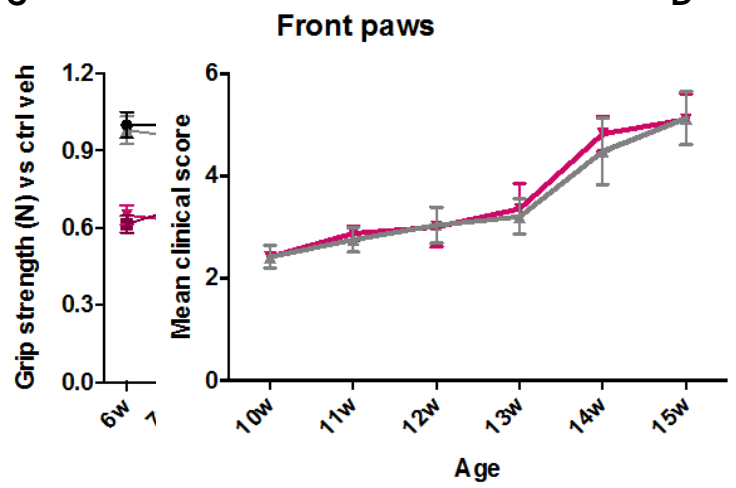

Front + hind paws

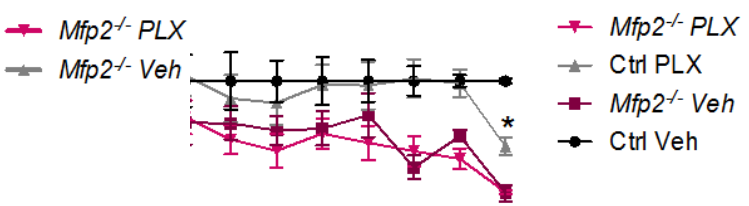

E

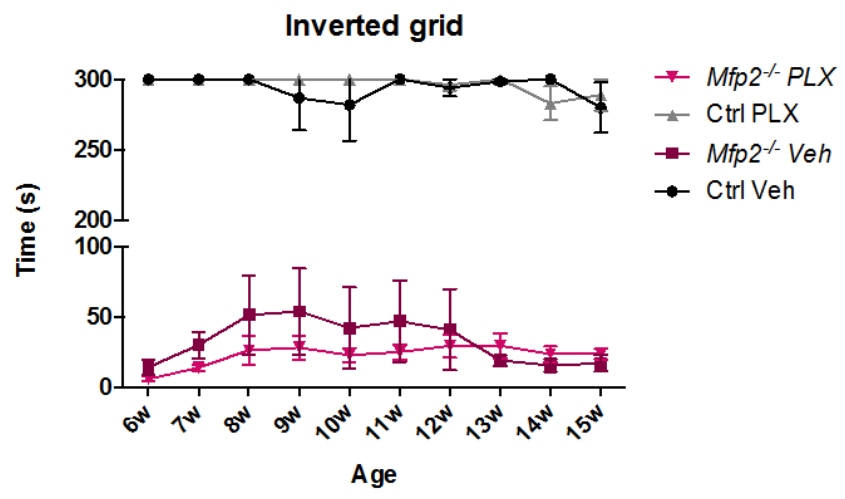


Figure 7

A

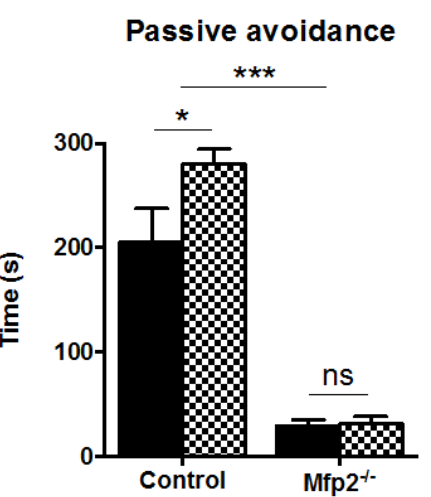

C

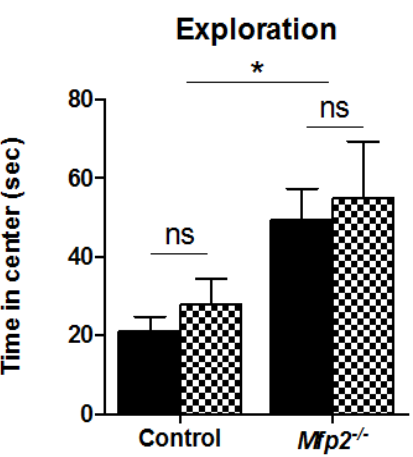

B

Locomotor activity

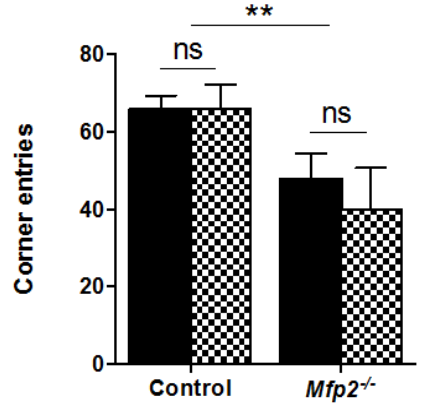

Vehicle \$ PLX
D

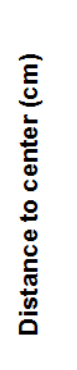

ns<smiles>C#CCCCCC</smiles>

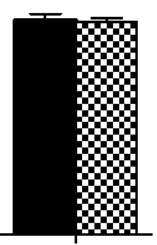

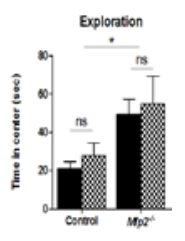

Control $\quad \mathrm{Mfp2}^{-/}$ 
Figure 8

A

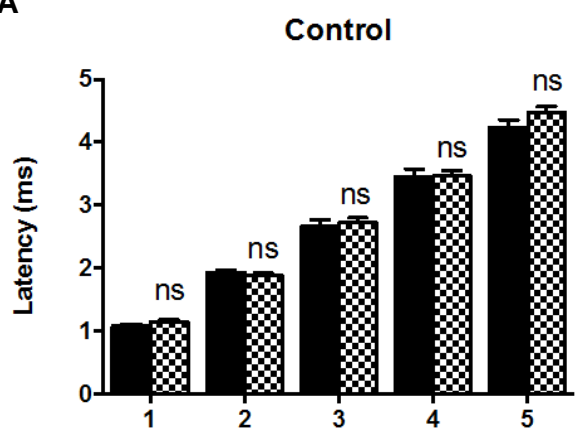

B

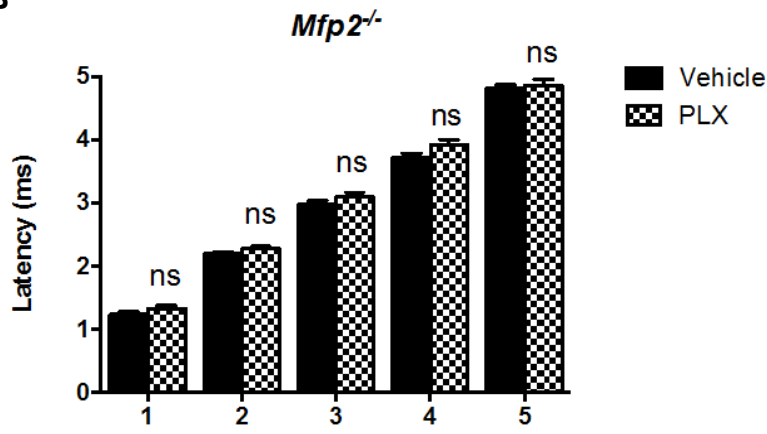

D

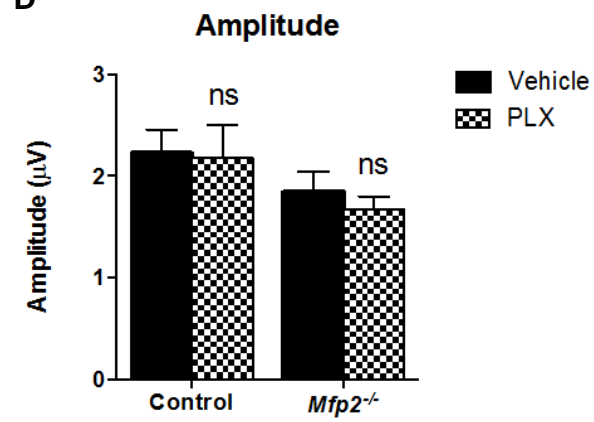

C

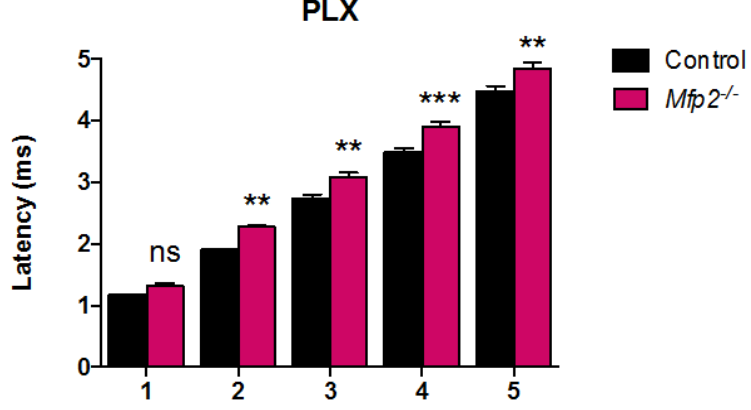

